\title{
The Design of Clothing Hanging System Based on Fisher Technik
}

\author{
Xiao Luo ${ }^{1, a}$, Yanping Wang ${ }^{2, b}$, Yi Liu ${ }^{3, c}$, Fei Guo ${ }^{4, d}$, Xiaojie Liu ${ }^{5, e}$ \\ 1,2,3,4,5 Department of Information Engineering, Beijing Institute of Fashion Technology, Beijing, \\ 100029, China
}

aemail: Ixiaorow@yeah.net

\begin{abstract}
Keywords: Fisher Technik; Clothing Hanging; Creative Combinations; Simulation
\end{abstract}
\begin{abstract}
Fischer Technik is a high-tech engineering assembled model. It is an ideal teaching aids for demonstrating scientific principles and technological processes. The main components of Fischer Technik are high quality nylon plastic with accurate dimensions; you can repeat disassembly without affecting the model combines accuracy. In this paper, Fischer model is designed as a garment hanging system, which uses a combination of computer-aided Fischer Technik components. The entire system is composed of a plurality of stations and applied throughout the entire production process. Every track interface is designed to automatically turn on and separated without causing blockage between each process, with efficient transport capacity. The system shows the real workflow garment hanging system and simulates normal pipeline operations. The whole process is effectively controlled and smooth.
\end{abstract}

\section{Background}

Clothing production is a kind of labor-intensive industry. In the past, completion of garment production all rely on manpower and the processes were cumbersome[1]. With the development of technology, post-production process is water-based operation, starting from the raw fabric, cutting, sampling, sewing and hot packs, each job requires a lot of workers to complete.

The traditional clothing assembly line is strapping production line. The clothing pieces are separated according to the type or number to a unit[2]. The workers pass to the different sewing workers according to the processing sequence, and so forth, until the whole process like sewing, ironing and lock button is finished. This model takes human workers more time, the single person only completes a process and it increases the auxiliary time. Location of equipment and station workers is relatively fixed, it is prone to the wrong piece of clothing pieces or miss the pieces. Once the production errors happen, rework rates will highly improve.

The clothing hanging system can change the production structure of the existing garment processing enterprise. Hanging system applies throughout the entire production process and connects every process[3]. Every track interface can be switched on and automatically separated without causing blockage between each process. The system can overcome the shortcomings of the traditional way, improve productivity and the workshop environment. In addition, hanging system can be put in parallel line. Thereby it can minimize the production cycle and improve the quality of business management.

Due to the size of the classroom and lab, modern teaching equipment in garment industry is relatively scarce. The students of clothing major can only learn the profession knowledge through books and pictures. To the clothing hanging system, it is difficult to understand each step without the concrete practice. Thus, we apply the Fischer Technik to design the clothing hanging system and get the good response.

\section{Fisher Technik}

In 1964, Fischer was born in Germany. It provides the best support for innovative education and experiments. Its dovetail patented design and creative industries combined model can make six-sided stitching and enable arbitrary combination and expansion[4]. Fischer technik includes the 
creative combination package, training models and industrial model. It covers the fields of mechanical, electronic, control, pneumatic, automotive technology, energy technology and robotics technology. Using the basic elements of industrial standards, supplemented by sensors, controllers, actuators and software complexes, you can achieve any reduction technology process and simulate the industrial production[5]. The Siemens, BMW, IBM and other US companies have adopted Fischer technik to demonstrate the production line.

\section{Clothing Hanging System}

The clothing hanging is a kind of flexible production management system. The basic constitution of this mode is a hanging object transport system. The transmission system has changed the binding type production mode of traditional garment industry[6]. It effectively solves the problems of production process such as the large auxiliary operation time ratio and long production period. Clothing hanging system is the production unit developed on CNC machine, robot, automatic warehouse and computer technology. The system can improve the utilization rate of equipment and production efficiency, reduce the processing auxiliary time and the semi-finished area. According to the man-machine operation mode, clothing hanging system consists of hanging clothes piece transmission device and sewing machinery controlled by computer. The basic principle of clothing hanging system is the whole pieces hanging on the racks of clothes, good processes based on prior input section, automatically sent to the next process in the hands of the operator. It greatly reduces the handling, binding, folding and other non-production time. When the production staff completes a process, hanging system will automatically be transferred to the next process station with simply pressing the control button. The whole procedure is as shown on figure 1:

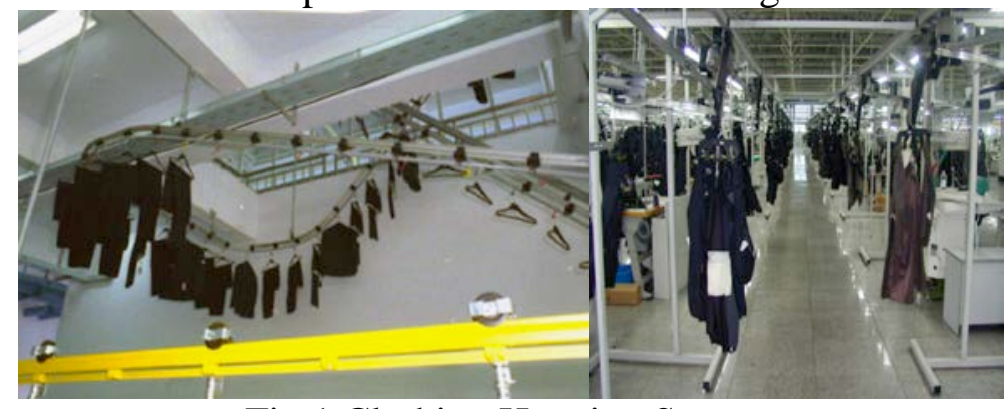

Fig.1 Clothing Hanging System

\section{The Design of Fisher hanging system}

The basic design idea of Fischer hanging system is hanging on the racks of clothes and automatically sending to the next operator based on sequence. The process flow diagram is shown on fig.2.

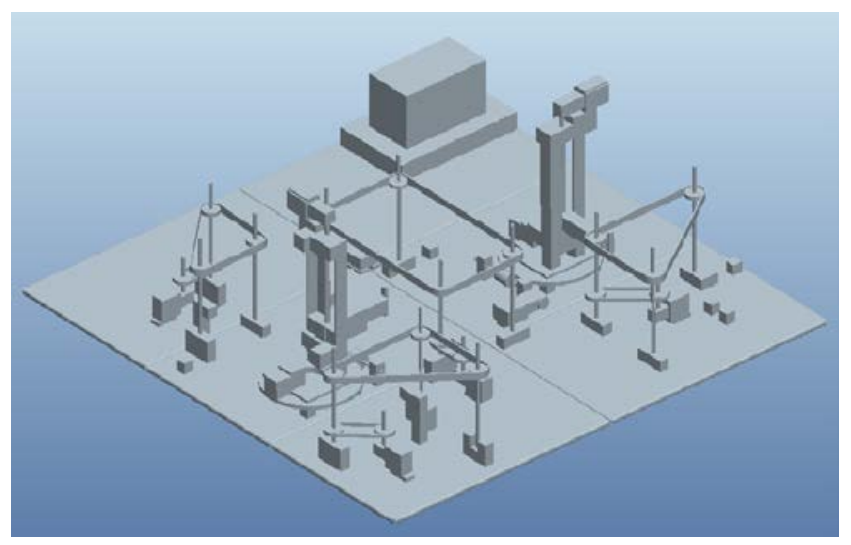

Fig.2 The overall simulation diagram

1. Hanging conveyor module

The system adopts Fischer technik components. The strutting piece hangs in midair and motor drives the gear. Through the gears are mutually engaged to drive the hanging above the rotary 
mechanism. We independently developed and fit the parts to form a suspension of transmission mechanism. The whole hanging system mechanism is divided into 5 individual operations of the ring. These five rings can be relatively independent movement and the synchronous state in the overall. As shown in figure 3.
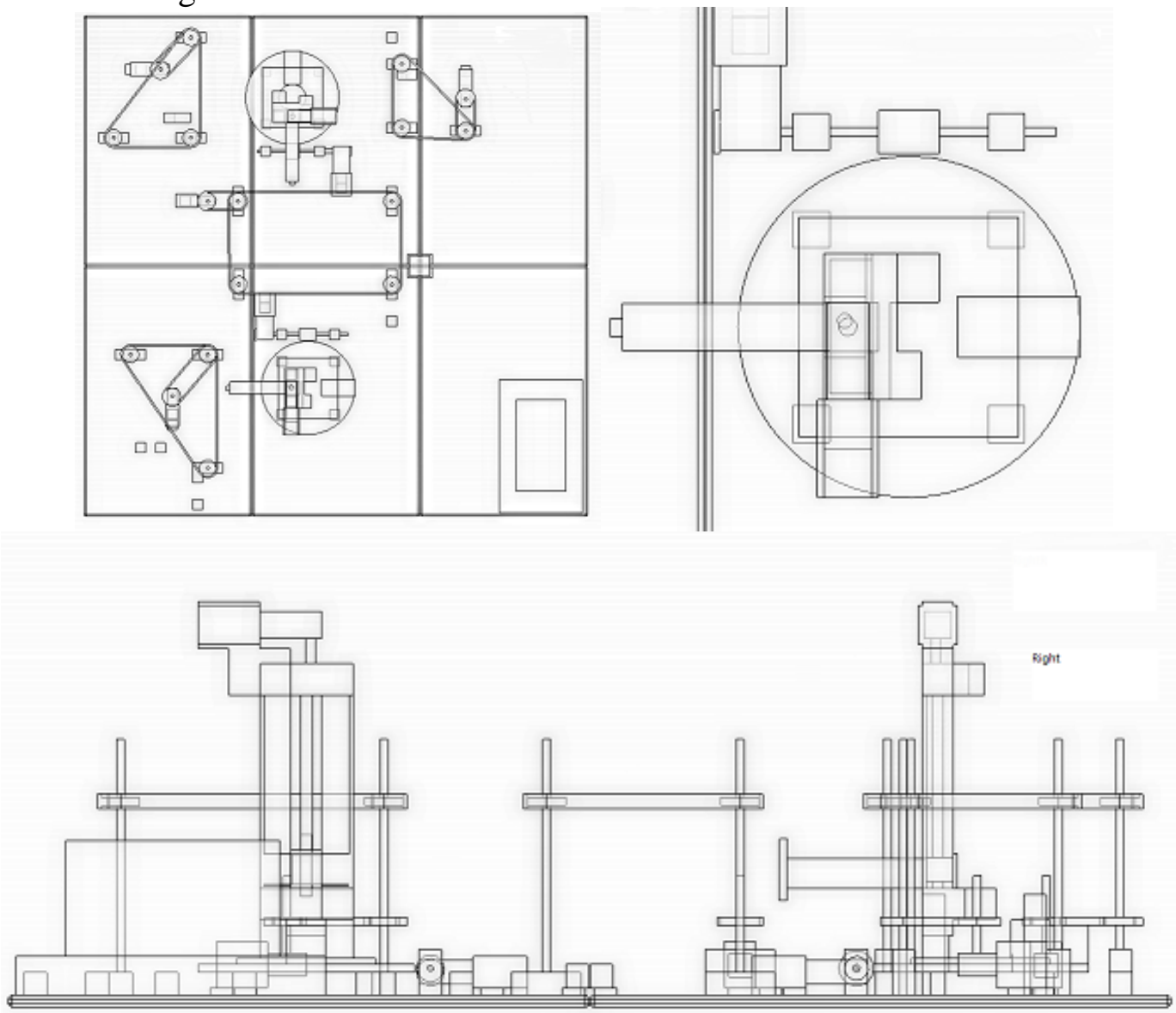

Fig.3 Assembly diagram

2. Transmission module

The Fischer technik is assembled into a free rotation, vertical lifting transmission device. Each independently parts of hanging system goes through the rotary table, it will trigger a light operated switch. And then the clothes is taken away and moved to the next processing ring, so back and forth, each of the parts under the hanging system complete through the five rings and become the finished garments. As shown in Fig. 4.

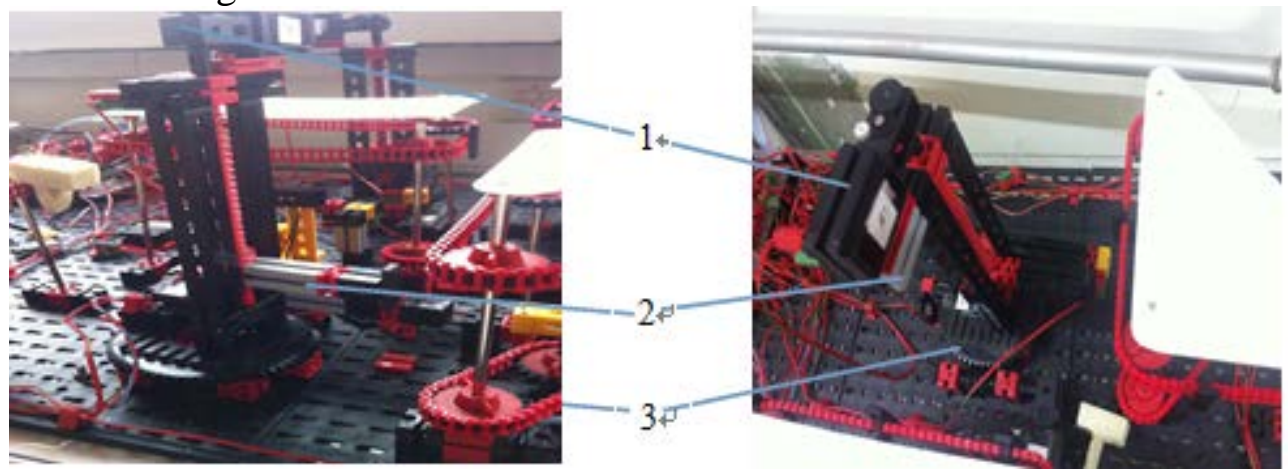

1. Motor 2. Suspension arm

3. Rotary table

Fig.4 Transmission module diagram

3. The positioning module

The system movement needs to locate, we choose Fischer technik light emitting source and the photosensitive element to complete the accurate positioning system.

4. The recognition module

By using the color sensor of Fischer technik, we can identify different processes of clothing. The 
color sensor identifies different colors through the reflected light. Different colors of clothes has the different reflectance spectra and the digital output corresponding. Therefore, it can be realized the function of identifying and distinguishing. The module flow chart is as follows fig.5.

\section{Conclusion}

The clothing hanging system model can acquire and repair data. It is convenient for the data analysis. Clothing hanging system for parallel production through flexible control workstation supplies stockpile and as far as possible to make production process management, so as to maximize shorten order production cycle, provide a cost-effective and efficient solution for the promotion of garment enterprises. In the process of module design, we realize the physical simulation of working process using the Fischer technik. At the same time, each module can be copied, to increase or decrease the number of stations, to achieve different production requirements. Clothing hanging system in this paper can help the students understand and master the garment production process and principle as much as possible. It has the very big role in promoting the level of the teaching activities and helping to master the practical operation method. In practical application, it also has well reflects.

\section{Acknowledgement}

In this paper, the research was sponsored by Beijing Institute of Fashion Technology Teaching reform and innovation team project (JGTD-1404) and Beijing Key Laboratory of Digital and Interactive Media Project (Project No. KF2013-13)( KF2013-01) and Training Project of Beijing Institute of Fashion Technology (PTTBIFT_YC_009).

\section{References}

[1] Yinhua Duan, Xi wu, Qinghui Wang; The design of the production line of flexible manufacturing based on Fischer technik model [J]; Journal of Chongqing University of Technology; 201204

[2] Tianjun Liu, Jianqiu Mao, Bohao Zhi; The design and production of obstacle avoidance of robot based on the "Fischer technik" [J]; Journal of Changzhou Institute of Technology;2012 02

[3] Ji Ding, Suolin Duan, Fuqiang Ren, Feng Jiang; The study of obstacle avoidance for wheeled mobile robot based on Fuzzy Planning [J]; Journal of Changzhou University (NATURAL SCIENCE EDITION); 201004

[4] Lei Han, Xuan Zheng, Xue Sun; The practice and development of Fisher Techink[J]; Mechanical and electrical technology; 201005

[5] Chengzhuo Wen, Xiwu, Qinghui Wang; Practice and application of Fisher based on LLWin control [J]; Research and exploration in laboratory; 201301

[6] Zhaojun Ma; Manipulator control based on improved fischer model [J]; Innovation and application of science and technology; 201230 